\title{
Dynamic Network Analysis Reveals Altered Temporal Variability in Brain Regions after Stroke: A Longitudinal Resting-State fMRI Study
}

\author{
Jianping $\mathrm{Hu}\left(\mathbb{D},{ }^{1,2}\right.$ Juan Du, ${ }^{3}$ Qiang Xu, ${ }^{4}$ Fang Yang, ${ }^{3}$ Fanyong Zeng, ${ }^{4}$ Yifei Weng, ${ }^{4}$ \\ Xi-jian Dai $\odot,{ }^{4}$ Rongfeng Qi, ${ }^{4}$ Xiaoxue Liu, ${ }^{4}$ Guangming $\mathrm{Lu}\left(\mathbb{1},{ }^{2,4,5}\right.$ and Zhiqiang Zhang $\oplus^{4,5}$ \\ ${ }^{1}$ Department of Radiology, The First Affiliated Hospital of Fujian Medical University, Fuzhou, China \\ ${ }^{2}$ Department of Medical Imaging, Jinling Hospital, Nanjing Clinical School, Southern Medical University, Nanjing, China \\ ${ }^{3}$ Department of Neurology, Jinling Hospital, Medical School of Nanjing University, Nanjing, China \\ ${ }^{4}$ Department of Medical Imaging, Jinling Hospital, Medical School of Nanjing University, China \\ ${ }^{5}$ State Key Laboratory of Analytical Chemistry for Life Science, Nanjing University, Nanjing, China
}

Correspondence should be addressed to Guangming Lu; cjr.luguangming@vip.163.com

and Zhiqiang Zhang; zhangzq2001@126.com

Received 14 June 2017; Revised 18 December 2017; Accepted 8 January 2018; Published 5 April 2018

Academic Editor: Wenhua Zheng

Copyright (C) 2018 Jianping Hu et al. This is an open access article distributed under the Creative Commons Attribution License, which permits unrestricted use, distribution, and reproduction in any medium, provided the original work is properly cited.

Recent fMRI studies have demonstrated that resting-state functional connectivity (FC) is of nonstationarity. Temporal variability of FC reflects the dynamic nature of brain activity. Exploring temporal variability of FC offers a new approach to investigate reorganization and integration of brain networks after stroke. Here, we examined longitudinal alterations of FC temporal variability in brain networks after stroke. Nineteen stroke patients underwent resting fMRI scans across the acute stage (withinone-week after stroke), subacute stage (within-two-weeks after stroke), and early chronic stage (3-4 months after stroke). Nineteen age- and sex-matched healthy individuals were enrolled. Compared with the controls, stroke patients exhibited reduced regional temporal variability during the acute stages, which was recovered at the following two stages. Compared with the acute stage, the subacute stage exhibited increased temporal variability in the primary motor, auditory, and visual cortices. Across the three stages, the temporal variability in the ipsilesional precentral gyrus (PreCG) was increased first and then reduced. Increased temporal variability in the ipsilesional PreCG from the acute stage to the subacute stage was correlated with motor recovery from the acute stage to the early chronic stage. Our results demonstrated that temporal variability of brain network might be a potential tool for evaluating and predicting motor recovery after stroke.

\section{Introduction}

Functional connectivity (FC) and functional connectomics based on resting-state functional magnetic resonance imaging (rs-fMRI) have proven to be powerful tools for investigating the brain function in both physiological and disease states [1-3]. Most of the studies on resting-state brain networks were based upon the assumption that the strength of FC is constant over scan session [4]. Recently, merging evidence suggests that the FC of the resting-state brain network is not static and presents temporal variability even within a session [5-7]. Temporal variation in FC, the so-called dynamic functional connectivity, can be attributed to neural activity $[7,8]$. Furthermore, disease-related alterations in the dynamic properties of FC have also been reported, suggesting that temporal features of FC could serve as a disease biomarker $[9,10]$.

Recently, a novel approach introduced by Zhang et al. could measure the temporal variability of functional architecture in a specific region, which is different from the conventional dynamic FC method which only measures the interregional property of FC variability [11]. The temporal variability of the particular brain region might reflect its dynamic reconfiguration into distinct functional regions 
TABle 1: Demographic and clinical data of stroke patients.

\begin{tabular}{lccc}
\hline Patients $(n=19)$ & Acute stage & Subacute stage & Early chronic stage \\
\hline Age (year) & $52.26 \pm 11.74(30-71)$ & - & - \\
Sex (male) & $17 / 19$ & - & - \\
Lesion side (left) & $12 / 19$ & - & - \\
Lesion volume (ml) & $3.26 \pm 1.93(0.61-6.44)$ & - & - \\
Days after stroke (day) & $4.11 \pm 1.76(1-7)$ & $10.47 \pm 2.12(8-14)$ & $99.32 \pm 8.54(87-116)$ \\
UL-FMA & $33.58 \pm 14.55(6-56)$ & $42.63 \pm 16.79(7-61)$ & $55.10 \pm 12.27(27-66)$ \\
\hline
\end{tabular}

UL-FMA: upper limb Fugl-Meyer assessment.

within the brain network at different times and might be an indicator of brain flexibility and adaptability [11]. Therefore, the temporal variability may provide new insight into the pathophysiological mechanisms of neural rehabilitation after brain damage such as the neural process of recovery after stroke.

Motor function impairment is one of the most common complications of stroke. Following initial cerebral damage, motor function in nearly all stroke patients can usually recover to some extent within the first six months after stroke [12]. However, the underlying mechanism of motor recovery after stroke is not entirely understood. A large number of fMRI studies have shown that motor recovery after stroke is a complicated process related to the cerebral structural and functional reorganization [13-17]. However, most of these studies have been confined to the exploration of static functional connectivity or networks, and few studies have carried out the approach of temporal variability.

In the present study, a longitudinal data of stroke patients with motor impairment across three consecutive stages was used to investigate the dynamic topological properties of brain networks by measuring the temporal variability of whole-brain functional networks. The first stage referred to as the acute stage was within the first week after symptom onset. The second stage referred to as the subacute stage was between one week and two weeks after the stroke onset. The third stage defined as the early chronic stage was at 3-4 months after the stroke onset [15]. Besides, we also introduced a group of age-matched healthy controls as the baseline. We sought to determine (1) how the temporal variability of brain functional network changes over the course of motor recovery after stroke and (2) whether the alterations of the regional temporal variability of brain functional network are associated with motor recovery. Exploring these issues could contribute to understanding the neurophysiological mechanisms for motor rehabilitation after stroke.

\section{Materials and Methods}

2.1. Participants. The local Ethical Committee approved the experiment, and all subjects signed the informed consent before the study. Nineteen patients (17 male, mean age $52.26 \pm 11.73$ years, 12 left-side deficits) with acute first-ever ischemic stroke were enrolled from January 2015 to March 2016. The inclusion criteria were as follows: (1) first-ever ischemic stroke, (2) unilateral hand motor deficit, (3) symptom onset $<7$ days, (4) age between 18 and 80 years, and (5) single stroke lesion located within the middle cerebral artery territory on MRI. The exclusion criteria consist of (1) hemorrhagic stroke, (2) bilateral stroke lesions on MRI, (3) language or cognitive deficits sufficient to affect informed consent, (4) other orthopedic, neurological, or psychiatric diseases substantially affecting the arm, (5) contraindications to MRI examination, and (6) recurred stroke during the follow-up. In addition, 19 healthy right-handed participants (12 men; 7 women; mean age $51.1 \pm 5.99$ years) were enrolled as the baseline.

Clinical measures and neuroimaging data were assessed at three consecutive time points during the poststroke phase. The mean time intervals between stroke onset and MRI scan points were as follows: the acute stage $(4.11 \pm 1.76$ days poststroke, ranging $1-7$ days), the subacute stage (10.47 \pm 2.12 days poststroke, ranging 8-14 days), and the early chronic stage (99.32 \pm 8.54 days poststroke, ranging 87-116 days). The lesion volumes were calculated at the acute stage. The mean lesion size was 3. $26 \pm 1.93 \mathrm{ml}$ (ranging 0.61-6.44 ml). The lesion map of each patient on the diffusion-weighted image was shown in Supplementary Figure 1. The hand motor function of each patient was assessed using an upper limb Fugl-Meyer Motor Assessment (UL-FMA), which has a range of 0 (complete hemiplegia) to 66 (normal performance) for upper extremities. The mean UL-FMA scores at three stages were as follows: $33.58 \pm 14.55$ (ranging 6-56) in the acute stage, $42.63 \pm 16.79$ (ranging 7-61) in the subacute stage, and $55.11 \pm 12.27$ (ranging 27-66) in the early chronic stage. Demographic and clinical characteristics of stroke patients were shown in Table 1 . The specific information of each patient was shown in Supplementary Table 1.

2.2. MRI Image Data Acquisition. All MR images were acquired using a $3.0 \mathrm{~T}$ whole-body scanner (Discovery MR750, GE Healthcare, Milwaukee, WI, USA) with a 32channel phased-array head coil. The participants were placed on the scanner gantry in a head-first supine position with a plastic holder to minimize head motion and earplugs to reduce scanner noise. High-resolution 3D T1-weighted structural images were obtained in the transverse orientation using a 3D-BRAVO sequence with the following parameters: $\mathrm{TR}=8.2 \mathrm{~ms}, \mathrm{TE}=3.2 \mathrm{~ms}$, flip angle $=12^{\circ}, \mathrm{FOV}=220 \mathrm{~mm} \times$ $220 \mathrm{~mm}$, matrix $=256 \times 256$, and slice thickness $=1.0 \mathrm{~mm}$. Functional MR images were acquired with a gradientecho EPI sequence with the following scan parameters: $\mathrm{TR}=2000 \mathrm{~ms}, \mathrm{TE}=30 \mathrm{~ms}$, flip angle $=80^{\circ}, \mathrm{FOV}=240 \mathrm{~mm} \times$ 


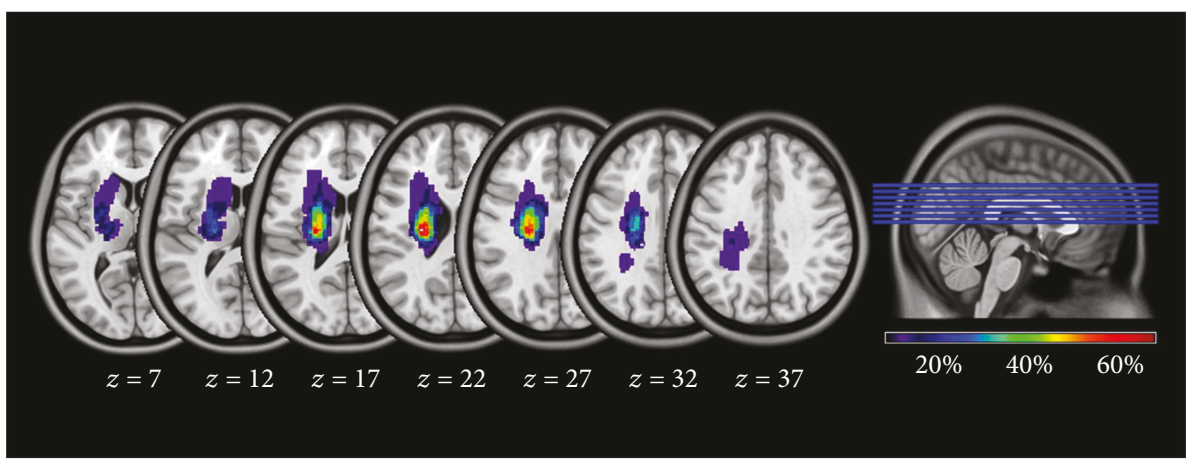

FIGURE 1: The overlap map of the lesions across all the stroke patients during the acute stage. Color bar indicates the percentage of the lesion overlap. $z$-axis from 7 to 37 in MNI coordinates. MNI: Montreal Neurological Institute.

$240 \mathrm{~mm}$, matrix $=64 \times 64$, slice thickness $=3.0 \mathrm{~mm}$, no gap, and slice number $=43$. The scan range covered the whole brain tissue from the vertex to the lower parts of the cerebellum. During the rs-fMRI scans, patients were instructed to keep their eyes closed, think of nothing in particular, and not fall asleep. Scan time lasted 6 minutes 50 second, and a total of 205 volumes were acquired.

2.3. fMRI Data Preprocessing. fMRI data preprocessing was conducted by SPM8 (http://www.fil.ion.ucl.ac.uk/spm) and Data Processing Assistant for Resting-State fMRI (DPARSF) [18]. Before data preprocessing, the imaging data of patients with right hemisphere lesions were flipped from the right to the left along the midsagittal plane. Thus, after flipping, the left hemisphere corresponded to the ipsilesional side and the right hemisphere corresponded to the contralesional side in all patients. The lesion over map across all patients in the acute stage was shown in Figure 1.

2.4. Process of Structural MRI Data. To avoid tissue misclassification led by infarcted tissue during segmentation and normalization, we used a cost function method to remove the influence of lesions $[19,20]$. The specific procedures were followed: firstly, the lesion mask was created on the individual 3D T1-weighted structural images by two radiologists (Hu JP and Zeng FY), with the guide of a DWI image. Secondly, a group-sample-specific brain template was generated. For each subject, the lesion-removed wholebrain mask was used as the cost function to normalize the 3D T1-weighted structural image into the standard brain template in MNI (Montreal Neurological Institute) space by using a 12-parameter affine transformation with nonlinear adjustments with $7 \times 8 \times 7$ basis functions. All individually normalized 3D T1-weighted structural images and all lesion-removed masks were averaged to yield a samplespecific brain template. Thirdly, the averaged 3D T1weighted structural template was segmented using the unified segment function of SPM8, with the averaged lesion-removed mask as the cost-function. Fourth, the individual space 3D T1-weighted structural images were segmented using unified segment function. The segment issues in the third step process were used as the templates for the current segment process, and the individual lesion-removed brain mask was used as the cost function. Finally, the segment parameters containing the affine transition matrix were generated, which would be used in the functional MRI image normalization.

2.5. Analysis of Resting-State Functional Image Data. Firstly, the first five volumes of each run were discarded to allow for the signal to reach equilibrium and for the patients to adapt to the scanning noise. The remaining 200 volumes were corrected for slice timing effects for each volume, and then all volumes were realigned to the first volume to adjust for the residual head movement. The estimated translation or rotation parameters did not exceed $1.5 \mathrm{~mm}$ or 1.5 degrees. Secondly, the realigned images were coregistered to the individual 3D T1-weighted images. Thirdly, the segment parameters gained in structural MRI data process were applied to the coregistered images for the normalization of functional images, and then the functional images were resampled to $3 \times 3 \times 3 \mathrm{~mm}^{3}$ voxel size. After normalization, the BOLD signal of each voxel in resting-state functional MR images was first detrended to abandon linear trend and then temporal band pass filter $(0.01-0.08 \mathrm{~Hz})$ was performed to reduce low-frequency drift and high-frequency physiological noise. Finally, sources of spurious variance, including head motion parameters and white matter, cerebrospinal, and global signals, were removed through linear regression.

2.6. Brain Regional Temporal Variability Analysis. The AAL (Automated Anatomical Labeling) template was used to parallel the brain into 116 ROIs (regions of interest). The time series of ROIs were gained by averaging the time series of all voxels in the corresponding ROIs [21, 22], and 116 regional time series were obtained for the later analysis. According to the steps described by Zhang et al. [11], the temporal variability of each brain region was calculated. The specific steps were as follows (Figure 2): firstly, all BOLD time series were segmented into $n$ nonoverlapping windows each with a window length $l$. Within the ith time window, the whole brain functional connectivity network $F_{i}$ (a $116 \times 116$ matrix, with 116 brain regions) was obtained using Pearson correlation analysis. Secondly, the functional architecture of a region $k$ at the $i$ th time window was defined as the overall functional connectivity profile of region $k$, which was a 116-dimensional vector and was shortened as 


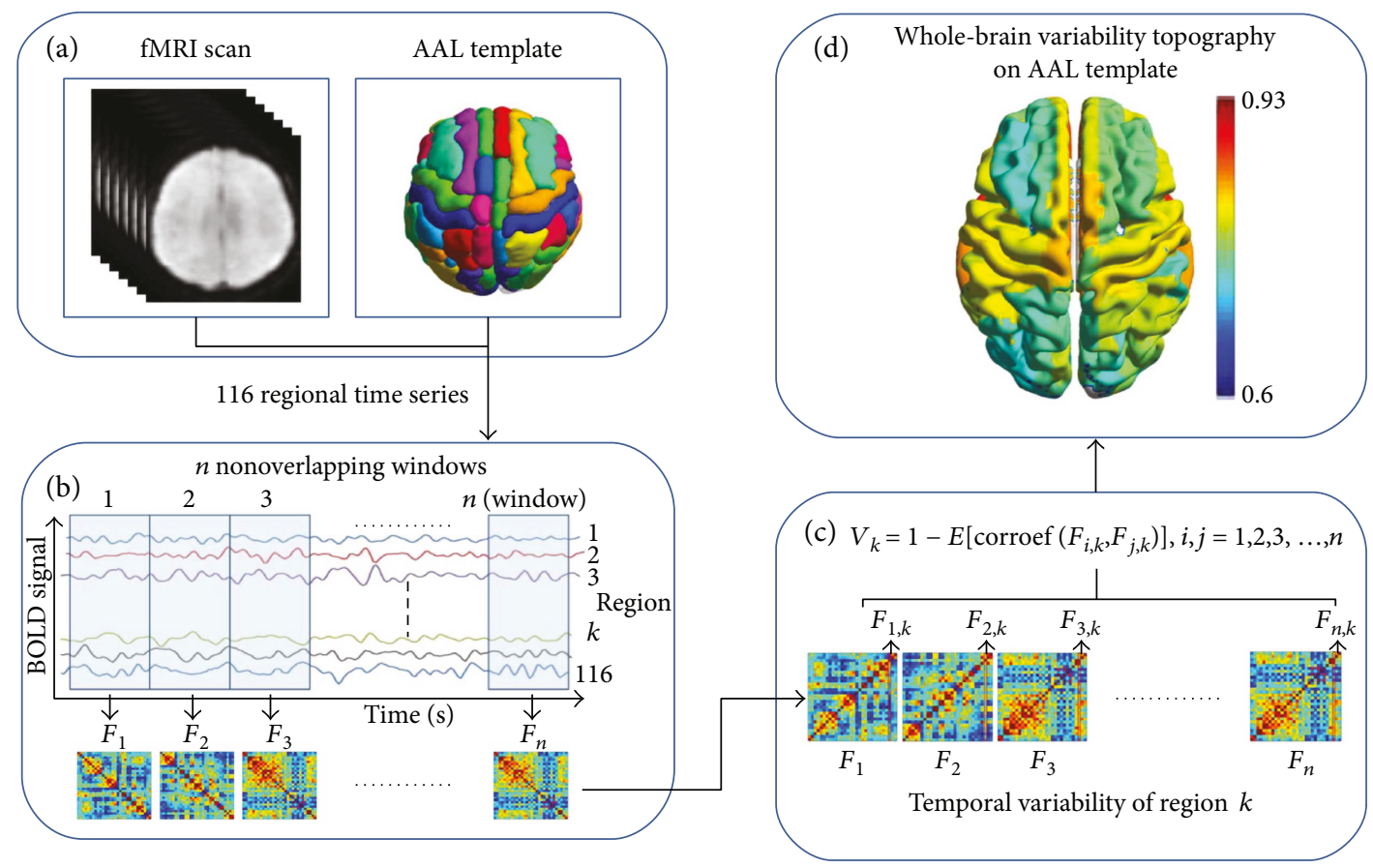

FIGURE 2: Definition of temporal variability for a given region $k$ in the functional network. (a) The BOLD time series for 116 brain regions were extracted from AAL template. (b) All BOLD time series were segmented into $n$ nonoverlapping windows. Functional networks are constructed in each time window. (c) The temporal variability of the region $k$ is determined by comparing functional connectivity profile of the region $k$ at different windows. (d) The topographic pattern of temporal variability in the whole brain based on the AAL template was demonstrated.

$F_{i, k}$. Thirdly, the temporal variability of a region of interest $k$ was computed by comparing the functional connectivity profile of region $k$ at different windows. The concrete formulation was listed as follows:

$$
V_{k}=1-\overline{\operatorname{corrcoef}\left(F_{i, k}, F_{j, k}\right)},
$$

where $i, j=1,2,3, \ldots, n, i \neq j$.

Here, $i, j$ meant different time windows and $n$ meant the number of total windows determined by window length $l$. Finally, to avoid the arbitrary choice of window length in the study, we calculated $V_{k}$ at different window lengths $l(l=10,11,12, \ldots, 20$ volumes $)$. The average value of all $V_{k}$ values over different window lengths was defined as the final temporal variability of the ROI.

2.7. Statistical Analysis. Statistical tests were performed using the MATLAB 2016a statistical package and SPSS version 22 (Statistical Package for the Social Sciences, IBM). Results are expressed as mean \pm standard deviation (SD). Two-sample $t$-test was used to investigate the significant difference in regional temporal variability of brain networks between the stroke patients and healthy controls. One-way repeated measures ANOVA and multiple comparisons (post hoc Tukey's test) was used to assess the significant differences in UL-FMA score and temporal variability in brain regions between the three stages of stroke progression.

To investigate the link between temporal variability in brain regions and motor performance over the course of motor recovery, Pearson correlation analysis was used to analyze the correlation between the change in ULFMA scores and the changes in temporal variability in brain regions with the significant difference between the three stages.

\section{Results}

3.1. Behavioral Data. One-way repeated measures ANOVA showed that the UL-FMA scores significantly increased over the process of motor recovery $(F=81.86, P<0.001)$, and post hoc test further indicated that there was a significant increase in UL-FMA scores between the three stages $(P<0.001)$ following motor recovery.

3.2. Differences in Temporal Variability of Brain Networks between Healthy Controls and Stroke Patients. The difference in temporal variability of brain networks was evaluated between healthy controls and stroke patients during three stages $(P<0.005$ with uncorrected, two sample $t$-test).

Compared with the healthy controls, the stroke patients exhibited reduced temporal variability in all brain regions showing significant changes during the three stages which were summarized in Table 2 and Figure 3 . The reduced temporal variability in brain regions covered the primary sensorimotor, auditory and visual cortices, and default mode network (DMN) which were found at the acute stage of the stroke group relative to healthy controls. Compared with the healthy controls, the stroke patients showed reduced temporal variability in ipsilesional postcentral 
TABLE 2: Differences in regional temporal variability between controls and stroke patients at three stages.

\begin{tabular}{|c|c|c|c|c|c|c|c|c|}
\hline \multicolumn{3}{|c|}{ Acute stage versus control } & \multicolumn{3}{|c|}{ Subacute stage versus control } & \multicolumn{3}{|c|}{ Early chronic stage versus control } \\
\hline Brain region & $T$ value & $P$ value & Brain region & $T$ value & $P$ value & Brain region & $T$ value & $P$ value \\
\hline \multicolumn{9}{|c|}{ The ipsilesional side } \\
\hline PreCG & -3.556 & $<0.001$ & PoCG & -3.025 & 0.005 & PreCG & -3.499 & 0.001 \\
\hline PoCG & -4.168 & $<0.001$ & ACG & -3.058 & 0.004 & PoCG & -3.423 & 0.002 \\
\hline PCL & -3.915 & $<0.001$ & CRBL45 & -3.158 & 0.003 & & & \\
\hline SMA & -4.056 & $<0.001$ & & & & & & \\
\hline SPG & -3.457 & $<0.001$ & & & & & & \\
\hline IPL & -3.572 & $<0.001$ & & & & & & \\
\hline ACG & -3.360 & 0.002 & & & & & & \\
\hline MCG & -3.367 & 0.002 & & & & & & \\
\hline Calcarine & -3.329 & 0.002 & & & & & & \\
\hline Cuneus & -3.067 & 0.004 & & & & & & \\
\hline ITG & -3.187 & 0.003 & & & & & & \\
\hline Insula & -3.262 & 0.002 & & & & & & \\
\hline FFG & -3.701 & $<0.001$ & & & & & & \\
\hline CRBL45 & -3.852 & $<0.001$ & & & & & & \\
\hline \multicolumn{9}{|c|}{ The contralesional side } \\
\hline PreCG & -3.309 & 0.002 & SFGdor & -3.079 & 0.004 & Hippocampus & -3.142 & 0.003 \\
\hline PoCG & -3.847 & $<0.001$ & Thalamus & -3.019 & 0.004 & & & \\
\hline SFGdor & -3.651 & $<0.001$ & & & & & & \\
\hline MFG & -3.211 & 0.003 & & & & & & \\
\hline ACG & -3.276 & 0.002 & & & & & & \\
\hline MCG & -3.201 & 0.003 & & & & & & \\
\hline MOG & -3.442 & $<0.001$ & & & & & & \\
\hline MTG & -3.769 & $<0.001$ & & & & & & \\
\hline FFG & -3.369 & 0.002 & & & & & & \\
\hline Thalamus & -3.347 & 0.002 & & & & & & \\
\hline CRBL45 & -3.775 & $<0.001$ & & & & & & \\
\hline
\end{tabular}

ACG: anterior cingulate and paracingulate gyri; MCG: median cingulate and paracingulate gyri; ITG: inferior temporal gyrus; FFG: fusiform gyrus; MFG: middle frontal gyrus; SFGdor: superior frontal gyrus, dorsolateral; MOG: middle occipital gyrus; MTG: middle temporal gyrus; PreCG: precental gyrus; PoCG: postcentral gyrus; PCL: paracentral lobule; SPG: superior parietal gyrus; IPL: inferior parietal lobule; SMA: supplementary motor area; CRBL45: cerebelum_4_5.

gyrus (PoCG), ipsilesional anterior cingulate gyri (ACG), ipsilesional cerebelum_4_5, contralesional superior parietal gyrus (SFG), and contralesional thalamus at the subacute stage. Reduced temporal variability in the ipsilesional precentral gyrus (PreCG), ipsilesional PoCG, and contralesional hippocampus was found at the early chronic stage of stroke patients relative to healthy controls.

\subsection{Longitudinal Changes in Temporal Variability of Brain} Networks over the Stages of Stroke. One-way repeated measures ANOVA showed that thirteen regions demonstrated significant differences in temporal variability among the three stages $(P<0.01$, Table 3, Figure 4). Multiple comparisons were further performed to estimate the differences in these brain regions (Table $3, P<0.05$ ). Compared to the acute stage, the subacute stage showed increased temporal variability in several brain regions such as the primary motor, auditory, and visual cortices and most of these brain regions kept increasing temporal variability at the early chronic stage. Compared to the subacute stage, the early chronic stage showed reduced temporal variability in the ipsilesional PreCG and the contralesional hippocampus while the other eleven brain regions demonstrated increased temporal variability.

3.4. The Relationships between Temporal Variability in Brain Regions and Motor Function. Motor recovery over the early chronic stage (increased UL-FMA from the acute stage to early chronic stage) significantly and positively correlated to change in temporal variability over the subacute stage (increased regional temporal variability from acute stage to subacute stage) in the ipsilesional PreCG $(r=0.67, P=$ 0.002 , Figure 5). Moreover, there was also no significant correlation between motor recovery and change in temporal variability in other brain regions over any other session interval. This finding indicates that long-term motor recovery is related to change in temporal variability in the ipsilesional PreCG over the subacute stage. 

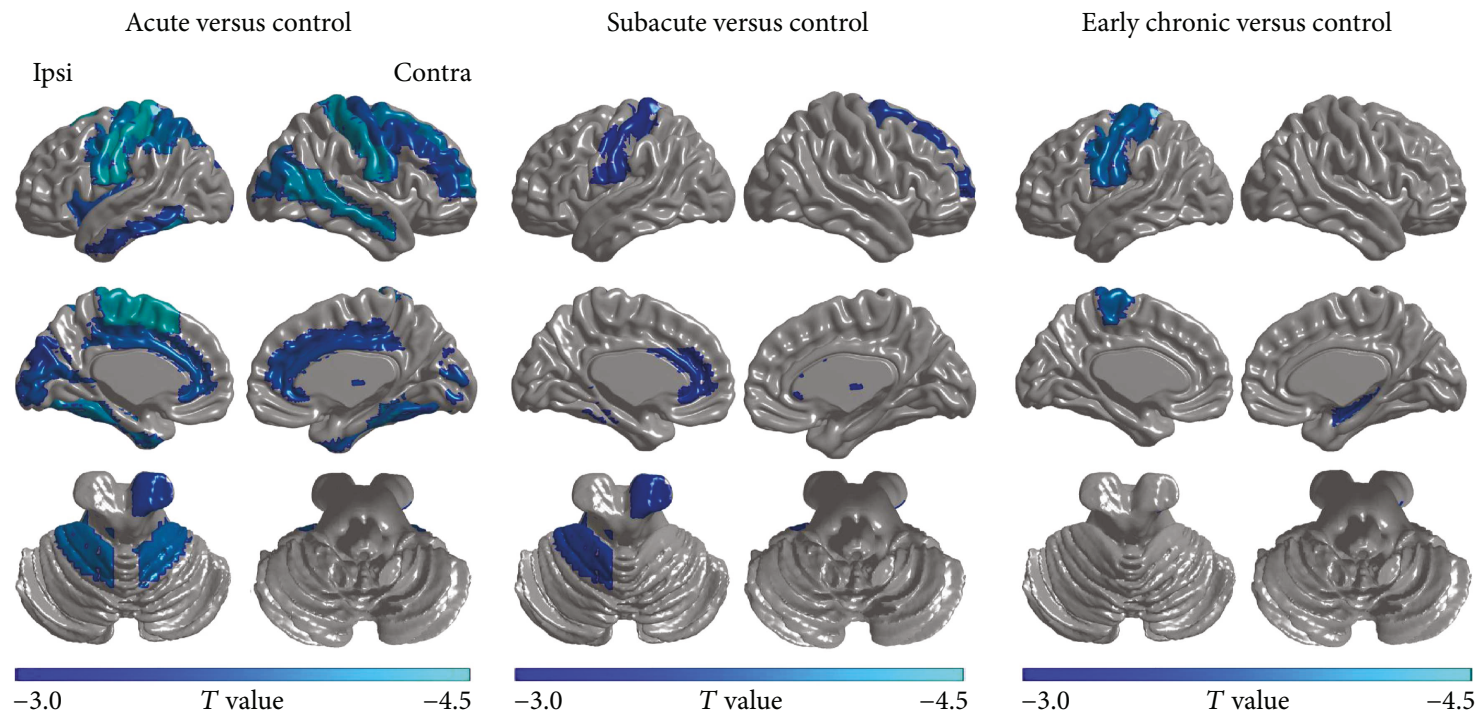

FIGURE 3: Brain regions showing significant differences in temporal variability between controls and patients during three stages (two-sample $t$-test, $P<0.005)$. All brain regions were summarized in Table 2. Ipsi: the ipsilesional side; Contra: the contralesional side.
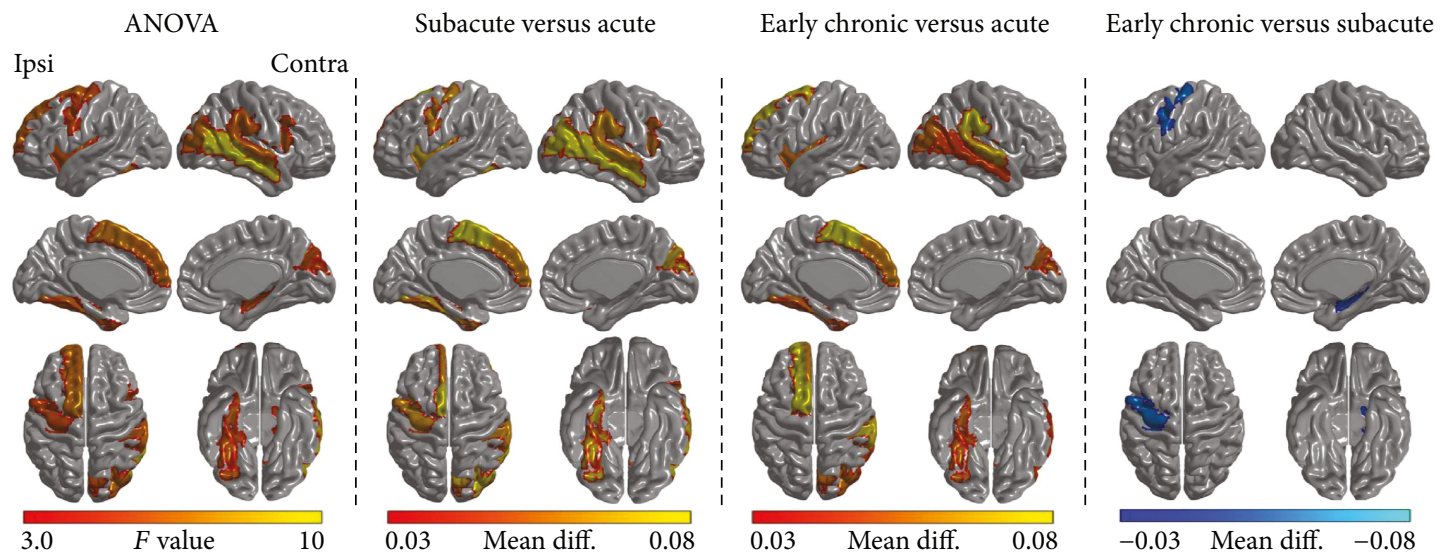

FIGURE 4: Brain regions showing significant differences in temporal variability across the three stages (one-way repeated measures ANOVA and post hoc test, $P<0.01$ and $P<0.05$, resp.). All brain regions were summarized in Table 3. Ipsi: the ipsilesional side; Contra: the contralesional side.

\section{Discussion}

In the present study, we investigated the longitudinal alteration in temporal variability of resting-state functional networks in stroke patients with motor function impairment. Our findings showed that (1) compared with healthy controls, stroke patients at the acute stage demonstrated extensively reduced temporal variability in several brain regions such as primary sensorimotor, auditory, visual cortices, and DMN. The temporal variability in these brain regions was restored to normal level from acute the stage to the early chronic stage except the ipsilesional sensorimotor cortex and contralesional hippocampus. (2) We observed a timedependent alteration in temporal variability of brain networks in the longitudinal study. Specifically, from the acute stage to the early chronic stage, the temporal variability in the ipsilesional PreCG and the contralesional hippocampus was increased first and then decreased, while the temporal variability of the other regions showed a trend of gradual increase. These findings suggest that the changes in the temporal variability may reflect dynamic reconfiguration of brain networks after stroke and may provide useful and complementary information for motor rehabilitation after stroke.

The temporal variability of a given brain region is considered to be negatively correlated to the variance of regional BOLD signal and energy of low-frequency component and to be positively correlated to the alpha-band oscillationrecorded electroencephalography [8]. Many studies have demonstrated that the slow-5 oscillations are particularly susceptible to disruption induced by the stroke. These studies showed that the stroke group exhibited an increasing trend in the low-frequency fluctuations at the acute stage $(<7$ days after stroke onset) and a decreasing trend from the acute stage to the early chronic stage, which may have been due to an inhibitory deficit from impaired the "task-positive" regions [23-25]. Besides, a decrease trend in alpha rhythm 
TABLE 3: Differences in regional temporal variability among three stages.

\begin{tabular}{|c|c|c|c|c|c|c|c|c|}
\hline \multirow{3}{*}{ Brain region } & \multirow{3}{*}{\multicolumn{2}{|c|}{$\begin{array}{c}\text { ANOVA } \\
F \text { value } \\
P \text { value }\end{array}$}} & \multicolumn{6}{|c|}{ Multiple comparisons (post hoc Tukey's test) } \\
\hline & & & \multicolumn{2}{|c|}{ Subacute versus acute } & \multicolumn{2}{|c|}{$\begin{array}{l}\text { Early chronic } \\
\text { versus acute }\end{array}$} & \multicolumn{2}{|c|}{$\begin{array}{c}\text { Early chronic } \\
\text { versus subacute }\end{array}$} \\
\hline & & & Mean diff. & $P$ value & Mean diff. & $P$ value & Mean diff. & $P$ value \\
\hline \multicolumn{9}{|c|}{ The ipsilesional side } \\
\hline PreCG & 5.288 & 0.009 & 0.067 & 0.0154 & - & - & -0.060 & 0.031 \\
\hline SMA & 7.003 & 0.003 & 0.083 & 0.011 & 0.0815 & 0.013 & - & - \\
\hline SFGmed & 6.377 & 0.004 & 0.064 & 0.013 & 0.063 & 0.015 & - & - \\
\hline Insula & 5.436 & 0.009 & 0.073 & 0.010 & 0.059 & 0.042 & - & - \\
\hline FFG & 6.458 & 0.004 & 0.074 & 0.005 & 0.059 & 0.026 & - & - \\
\hline SFGdor & 6.575 & 0.004 & - & - & 0.081 & 0.003 & - & - \\
\hline \multicolumn{9}{|c|}{ The contralesional side } \\
\hline MTG & 10.829 & $<0.001$ & 0.084 & $<0.001$ & 0.046 & 0.041 & - & - \\
\hline STG & 6.179 & 0.005 & 0.064 & 0.02 & 0.073 & 0.007 & - & - \\
\hline SMG & 6.018 & 0.006 & 0.066 & 0.030 & 0.081 & 0.007 & - & - \\
\hline MOG & 6.795 & 0.003 & 0.083 & 0.002 & 0.060 & 0.037 & - & - \\
\hline Cuneus & 5.460 & 0.008 & 0.073 & 0.009 & 0.058 & 0.044 & - & - \\
\hline IFGoperc & 5.965 & 0.006 & 0.062 & 0.034 & - & - & - & - \\
\hline Hippocampus & 5.638 & 0.007 & - & - & - & - & -0.053 & 0.007 \\
\hline
\end{tabular}

Mean diff.: mean difference; PreCG: precental gyrus; SMA: supplementary motor area; SFGmed: superior frontal gyrus, medial; FFG: fusiform gyrus; SFGdor: superior frontal gyrus, dorsolateral; STG: superior temporal gyrus; SMG: supramarginal gyrus; MOG: middle occipital gyrus; IFGoperc: inferior frontal gyrus, opercular part.

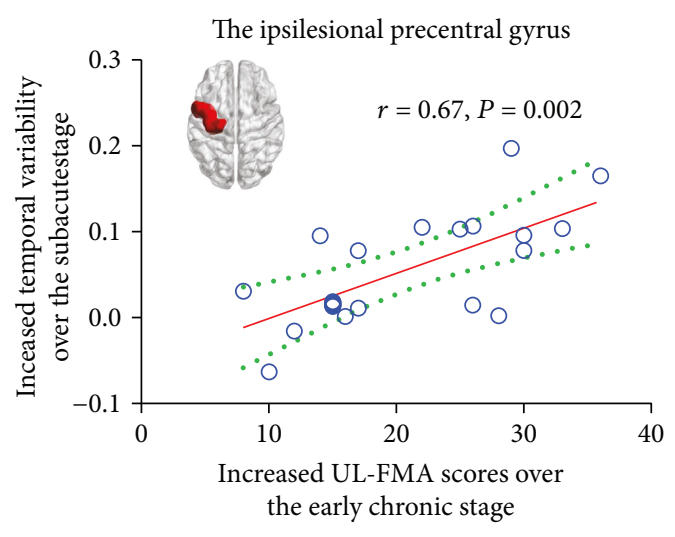

FIGURE 5: Correlation result between altered temporal variability and motor recovery (Pearson correlation analysis, $P<0.05$ ). The increased temporal variability of the ipsilesional precentral gyrus over the subacute stage was positively correlated to the increased UL-FMA over the early chronic stage.

both in acute and in subacute stroke patients has been reported in EEG studies [26-28]. In our results, when compared to healthy controls, stroke patients exhibited reduced temporal variability in all brain regions showing significant changes during the acute stage. On the basis of these previous studies, we discreetly speculate that the disruption of function network integrity and the damage of regional neural activity induced by stroke could be responsible for the reduced temporal variability in brain regions after stroke, especially at the acute stage. Moreover, previous studies have indicated that the increase in BOLD signal variability is beneficial to more efficiently process and respond to unexpected external events $[29,30]$, and brain regions with more lowfrequency components tend to be more easy to synchronize with other regions $[11,31]$. At such condition, keeping low temporal variability in functional networks is also beneficial to maintain information transmission and functional integration with other brain regions.

In the longitudinal study, we found that temporal variability in brain regions of multiple functional networks, including DMN, attention network, and visual network, significantly increased and restored to normal level when compared with the healthy controls during the subacute stages. In line with these findings, several studies have pointed out that stroke recovery might involve the alternations of resting-state networks (RSNs), involving inter-RSNs and intra-RSNs [4, 32-34]. The temporal variability associated with a brain region might reflect its dynamical reconfiguration into distinct brain regions within the brain network [8]. Such an increase in temporal variability during the subacute stage may well be validated and explicated by many studies on stroke, in which excessive neuronal clustering and wiring were observed in the peri-infarct regions at the initial recovery phase. For example, previous studies have demonstrated that new structural circuits in both the perilesional zone and other distant regions would reestablish to compensate for the loss of function in the damaged cortex during the process of functional reorganization after stroke. What is more is that branch-specific remodeling of the dendritic arbor in the peri-infarct cortex dramatically increased within the first two weeks after stroke and is still evident six weeks after stroke $[35,36]$. A brain network study based on animals also found that at the initial phases of poststroke recovery, neural spine elongation, neurite sprouting, might create a state of over 
connectivity that leads to the loss of signal synchronization and increase in random integration between neurons, resulting in reduced cortical signal coherence and FC [37]. Collectively, our findings suggest that rapidly increasing temporal variability in the related brain regions is an important feature in the reorganization and integration of resting-state functional network during the subacute stage.

Our results also demonstrated reduced temporal variability in the ipsilesional PreCG and the contralesional hippocampus during the early chronic stage. Reduced temporal variability within a given region means that communication between this region and the other regions remains in a high degree of synchronization across different time windows, which is consistent with increased FC between this region and other regions. These findings were in line with previous studies, in which the FC strength restoration of the ipsilesional PreCG with other brain regions was an essential feature during motor recovery after stroke [38]. Remarkably, the contralesional hippocampus, which usually exhibits high temporal variability in healthy subjects [11], showed reduced temporal variability during the early chronic stage. Although the underlying mechanism remains unclear, functional and structural alterations in the cognitive-related brain regions such as the hippocampus have been extensively reported to facilitate recovery of motor function in stroke patients [3941]. In particular, reduced temporal variability in the contralesional hippocampus may be related to its increased gray matter volume [39].

A number of fMRI studies have demonstrated that early fMRI brain activation patterns were associated with subsequent motor recovery independent of initial motor impairment [42-45]. In the present study, we observed that increased temporal variability of the ipsilesional precentral gyrus over the subacute stage was positively correlated with the long-term motor recovery. Previous studies have pointed out that the reinstatement of previously reduced neural activity in the ipsilesional primary motor areas was the important feature of motor recovery after stroke [16, 42, 46-48]. Our result further strengthened the understanding for the important role played by the ipsilesional primary motor cortex in motor recovery after stroke and also indicated that altered temporal variability of the ipsilesional precentral gyrus might serve as a prediction indicator in motor recovery after stroke.

There are some limitations in the present study. Firstly, although we restricted the participants to stroke patients with single stroke lesion located within the middle cerebral artery territory, the location and size of stroke lesion exhibited a relative heterogeneity across the patients, which could create challenges in result interpretation. Secondly, although we adopted a longitudinal study design, three months follow-up with three time points is relatively short. A longer follow-up period with more time points is helpful to fully understand the relationship between temporal variability of functional network and stroke recovery in the future study. Finally, the impact of flipping image data on results is unclear.

In summary, using the dynamic network analysis, we presented a time-dependent topological pattern in temporal variability of brain network in stroke patients with motor impairment. We also demonstrated the tight relationship between altered temporal variability of the ipsilesional precentral gyrus and motor recovery after stroke. Although the study was preliminary and in a modest sample size, our results support that measuring the temporal variability of brain network may be a potential tool for evaluating and predicting the motor recovery after stroke. These findings expand our understanding of the dynamic properties of brain networks and provide new insight into the underlying mechanisms of reorganization and integration of brain network over the recovery process after stroke.

\section{Conflicts of Interest}

The authors declare no conflict of interest.

\section{Authors' Contributions}

Jianping $\mathrm{Hu}$ and Juan Du contributed equally to this work.

\section{Acknowledgments}

This study was supported by the special scientific research fund of public welfare profession of the National Health and Family Planning Commission of the People's Republic of China (201402019), Jiangsu Natural Science Foundation (BK20141373), Independent research project in the State Key Laboratory of Analytical Chemistry for Life Science (5431ZZXM1716), and National Natural Science Foundation of China (Grant no. 81701678). The authors gratefully acknowledge Yong Zhang (GE healthcare) for his excellent MR technique support.

\section{Supplementary Materials}

Table 1: demographic and clinical data of stroke patients. Figure 1: the lesion locations of the stroke patients. The lesions are shown on axial slices of the diffusion-weighted images. (Supplementary Materials)

\section{References}

[1] R. L. Buckner, F. M. Krienen, and B. T. T. Yeo, "Opportunities and limitations of intrinsic functional connectivity MRI," Nature Neuroscience, vol. 16, no. 7, pp. 832-837, 2013.

[2] B. Guerra-Carrillo, A. P. Mackey, and S. A. Bunge, "Restingstate fMRI: a window into human brain plasticity," The Neuroscientist, vol. 20, no. 5, pp. 522-533, 2014.

[3] S. M. Smith, D. Vidaurre, C. F. Beckmann et al., "Functional connectomics from resting-state fMRI," Trends in Cognitive Sciences, vol. 17, no. 12, pp. 666-682, 2013.

[4] C. Wang, W. Qin, J. Zhang et al., "Altered functional organization within and between resting-state networks in chronic subcortical infarction," Journal of Cerebral Blood Flow \& Metabolism, vol. 34, no. 4, pp. 597-605, 2014.

[5] R. Hindriks, M. H. Adhikari, Y. Murayama et al., "Can slidingwindow correlations reveal dynamic functional connectivity in resting-state fMRI?," NeuroImage, vol. 127, pp. 242-256, 2016. 
[6] R. M. Hutchison, T. Womelsdorf, E. A. Allen et al., "Dynamic functional connectivity: promise, issues, and interpretations," NeuroImage, vol. 80, pp. 360-378, 2013.

[7] C. Chang, Z. Liu, M. C. Chen, X. Liu, and J. H. Duyn, "EEG correlates of time-varying BOLD functional connectivity," NeuroImage, vol. 72, pp. 227-236, 2013.

[8] G. J. Thompson, M. D. Merritt, W. J. Pan et al., "Neural correlates of time-varying functional connectivity in the rat," NeuroImage, vol. 83, pp. 826-836, 2013.

[9] F. Liu, Y. Wang, M. Li et al., "Dynamic functional network connectivity in idiopathic generalized epilepsy with generalized tonic-clonic seizure," Human Brain Mapping, vol. 38, no. 2, pp. 957-973, 2017.

[10] E. Damaraju, E. A. Allen, A. Belger et al., "Dynamic functional connectivity analysis reveals transient states of dysconnectivity in schizophrenia," Neuroimage:Clinical, vol. 5, pp. 298-308, 2014.

[11] J. Zhang, W. Cheng, Z. Liu et al., "Neural, electrophysiological and anatomical basis of brain-network variability and its characteristic changes in mental disorders," Brain, vol. 139, no. 8, pp. 2307-2321, 2016.

[12] J. Ludemann-Podubecka, K. Bösl, S. Theilig, R. Wiederer, and D. A. Nowak, "The effectiveness of $1 \mathrm{~Hz}$ rTMS over the primary motor area of the unaffected hemisphere to improve hand function after stroke depends on hemispheric dominance," Brain Stimulation, vol. 8, no. 4, pp. 823-830, 2015.

[13] M. Veldsman, T. Cumming, and A. Brodtmann, "Beyond BOLD: optimizing functional imaging in stroke populations," Human Brain Mapping, vol. 36, no. 4, pp. 1620-1636, 2015.

[14] C. Grefkes and N. S. Ward, "Cortical reorganization after stroke: how much and how functional?," The Neuroscientist, vol. 20, no. 1, pp. 56-70, 2014.

[15] E. B. Plow, D. A. Cunningham, N. Varnerin, and A. Machado, "Rethinking stimulation of the brain in stroke rehabilitation: why higher motor areas might be better alternatives for patients with greater impairments," The Neuroscientist, vol. 21, no. 3, pp. 225-240, 2015.

[16] J. Zhang, L. Meng, W. Qin, N. Liu, F. D. Shi, and C. Yu, "Structural damage and functional reorganization in ipsilesional $\mathrm{ml}$ in well-recovered patients with subcortical stroke," Stroke, vol. 45, no. 3, pp. 788-793, 2014.

[17] L. Wang, C. Yu, H. Chen et al., "Dynamic functional reorganization of the motor execution network after stroke," Brain, vol. 133, no. 4, pp. 1224-1238, 2010.

[18] Y. Chao-Gan and Z. Yu-Feng, "DPARSF: a MATLAB toolbox for "pipeline" data analysis of resting-state fMRI," Frontiers in Systems Neuroscience, vol. 4, p. 13, 2010.

[19] G. T. Stebbins, D. L. Nyenhuis, C. Wang et al., "Gray matter atrophy in patients with ischemic stroke with cognitive impairment," Stroke, vol. 39, no. 3, pp. 785-793, 2008.

[20] W. Wei, Z. Zhang, Q. Xu, F. Yang, K. Sun, and G. Lu, "More severe extratemporal damages in mesial temporal lobe epilepsy with hippocampal sclerosis than that with other lesions: a multimodality MRI study," Medicine, vol. 95, no. 10, article e3020, 2016.

[21] N. Tzourio-Mazoyer, B. Landeau, D. Papathanassiou et al., "Automated anatomical labeling of activations in SPM using a macroscopic anatomical parcellation of the MNI MRI single-subject brain," NeuroImage, vol. 15, no. 1, pp. 273$289,2002$.
[22] Z. Zhang, W. Liao, H. Chen et al., "Altered functional-structural coupling of large-scale brain networks in idiopathic generalized epilepsy," Brain, vol. 134, no. 10, pp. 2912-2928, 2011.

[23] Q. L. Yao, H. Y. Zhang, B. B. Nie, F. Fang, Y. Jiao, and G. J. Teng, "MRI assessment of amplitude of low-frequency fluctuation in rat brains with acute cerebral ischemic stroke," Neuroscience Letters, vol. 509, no. 1, pp. 22-26, 2012.

[24] C. La, V. A. Nair, P. Mossahebi et al., "Implication of the slow5 oscillations in the disruption of the default-mode network in healthy aging and stroke," Brain Connectivity, vol. 6, no. 6, pp. 482-495, 2016.

[25] C. La, P. Mossahebi, V. A. Nair et al., "Differing patterns of altered Slow-5 oscillations in healthy aging and ischemic stroke," Frontiers in Human Neuroscience, vol. 10, p. 156, 2016.

[26] S. P. Finnigan, M. Walsh, S. E. Rose, and J. B. Chalk, "Quantitative EEG indices of sub-acute ischaemic stroke correlate with clinical outcomes," Clinical Neurophysiology, vol. 118, no. 11, pp. 2525-2532, 2007.

[27] S. Dubovik, J. M. Pignat, R. Ptak et al., "The behavioral significance of coherent resting-state oscillations after stroke," NeuroImage, vol. 61, no. 1, pp. 249-257, 2012.

[28] F. Zappasodi, E. Olejarczyk, L. Marzetti, G. Assenza, V. Pizzella, and F. Tecchio, "Fractal dimension of EEG activity senses neuronal impairment in acute stroke," PLoS One, vol. 9, no. 6, article e100199, 2014.

[29] D. D. Garrett, N. Kovacevic, A. R. McIntosh, and C. L. Grady, "Blood oxygen level-dependent signal variability is more than just noise," The Journal of Neuroscience, vol. 30, no. 14, pp. 4914-4921, 2010.

[30] D. D. Garrett, N. Kovacevic, A. R. McIntosh, and C. L. Grady, "The modulation of BOLD variability between cognitive states varies by age and processing speed," Cerebral Cortex, vol. 23, no. 3, pp. 684-693, 2013.

[31] G. Buzsaki and A. Draguhn, "Neuronal oscillations in cortical networks," Science, vol. 304, no. 5679, pp. 1926-1929, 2004.

[32] S. Ovadia-Caro, K. Villringer, J. Fiebach et al., "Longitudinal effects of lesions on functional networks after stroke," Journal of Cerebral Blood Flow \& Metabolism, vol. 33, no. 8, pp. 1279$1285,2013$.

[33] S. Lassalle-Lagadec, I. Sibon, B. Dilharreguy, P. Renou, O. Fleury, and M. Allard, "Subacute default mode network dysfunction in the prediction of post-stroke depression severity," Radiology, vol. 264, no. 1, pp. 218-224, 2012.

[34] A. M. Golestani, S. Tymchuk, A. Demchuk, B. G. Goodyear, and VISION-2 Study Group, "Longitudinal evaluation of resting-state FMRI after acute stroke with hemiparesis," Neurorehabilitation and Neural Repair, vol. 27, no. 2, pp. 153163, 2013.

[35] C. E. Brown, P. Li, J. D. Boyd, K. R. Delaney, and T. H. Murphy, "Extensive turnover of dendritic spines and vascular remodeling in cortical tissues recovering from stroke," Journal of Neuroscience, vol. 27, no. 15, pp. 4101-4109, 2007.

[36] C. E. Brown, J. D. Boyd, and T. H. Murphy, "Longitudinal in vivo imaging reveals balanced and branch-specific remodeling of mature cortical pyramidal dendritic arbors after stroke," Journal of Cerebral Blood Flow \& Metabolism, vol. 30, no. 4, pp. 783-791, 2010.

[37] M. P. van Meer, W. M. Otte, K. van der Marel et al., "Extent of bilateral neuronal network reorganization and functional recovery in relation to stroke severity," The Journal of Neuroscience, vol. 32, no. 13, pp. 4495-4507, 2012. 
[38] A. K. Rehme and C. Grefkes, "Cerebral network disorders after stroke: evidence from imaging-based connectivity analyses of active and resting brain states in humans," The Journal of Physiology, vol. 591, no. 1, pp. 17-31, 2013.

[39] F. Fan, C. Zhu, H. Chen et al., "Dynamic brain structural changes after left hemisphere subcortical stroke," Human Brain Mapping, vol. 34, no. 8, pp. 1872-1881, 2013.

[40] L. V. Gauthier, E. Taub, C. Perkins, M. Ortmann, V. W. Mark, and G. Uswatte, "Remodeling the brain: plastic structural brain changes produced by different motor therapies after stroke," Stroke, vol. 39, no. 5, pp. 1520-1525, 2008.

[41] W. Li, T. Han, W. Qin et al., "Altered functional connectivity of cognitive-related cerebellar subregions in well-recovered stroke patients," Neural Plasticity, vol. 2013, Article ID 452439, 10 pages, 2013.

[42] A. K. Rehme, L. J. Volz, D. L. Feis, S. B. Eickhoff, G. R. Fink, and C. Grefkes, "Individual prediction of chronic motor outcome in the acute post-stroke stage: behavioral parameters versus functional imaging," Human Brain Mapping, vol. 36, no. 11, pp. 4553-4565, 2015.

[43] S. C. Cramer, T. B. Parrish, R. M. Levy et al., "Predicting functional gains in a stroke trial," Stroke, vol. 38, no. 7, pp. 21082114, 2007.

[44] R. S. Marshall, E. Zarahn, L. Alon, B. Minzer, R. M. Lazar, and J. W. Krakauer, "Early imaging correlates of subsequent motor recovery after stroke," Annals of Neurology, vol. 65, no. 5, pp. 596-602, 2009.

[45] E. Zarahn, L. Alon, S. L. Ryan et al., "Prediction of motor recovery using initial impairment and fMRI $48 \mathrm{~h}$ poststroke," Cerebral Cortex, vol. 21, no. 12, pp. 2712-2721, 2011.

[46] A. K. Rehme, G. R. Fink, D. Y. von Cramon, and C. Grefkes, "The role of the contralesional motor cortex for motor recovery in the early days after stroke assessed with longitudinal FMRI," Cerebral Cortex, vol. 21, no. 4, pp. 756-768, 2011.

[47] A. K. Rehme, S. B. Eickhoff, L. E. Wang, G. R. Fink, and C. Grefkes, "Dynamic causal modeling of cortical activity from the acute to the chronic stage after stroke," NeuroImage, vol. 55, no. 3, pp. 1147-1158, 2011.

[48] I. Favre, T. A. Zeffiro, O. Detante, A. Krainik, M. Hommel, and A. Jaillard, "Upper limb recovery after stroke is associated with ipsilesional primary motor cortical activity: a meta-analysis," Stroke, vol. 45, no. 4, pp. 1077-1083, 2014. 


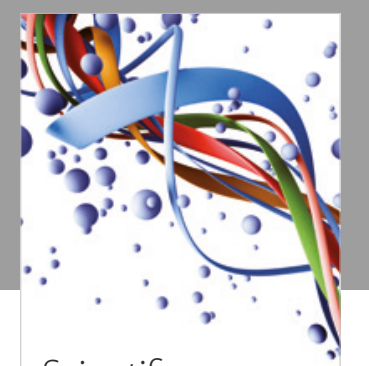

Scientifica
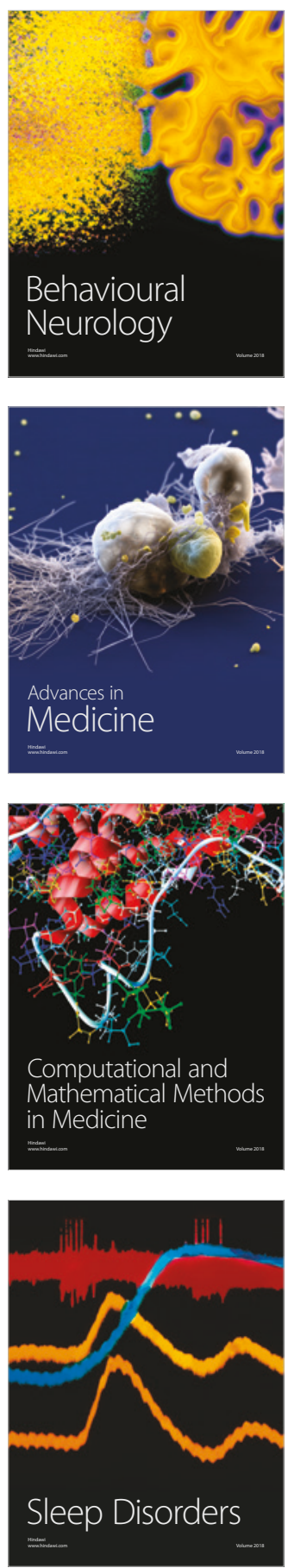

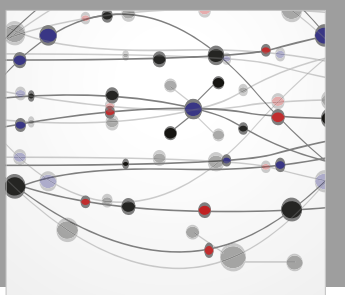

The Scientific World Journal

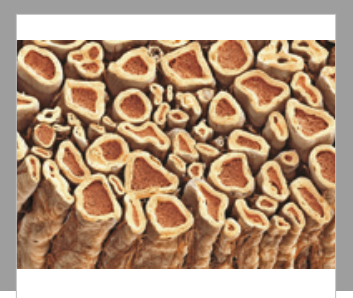

Case Reports in

Neurological Medicine

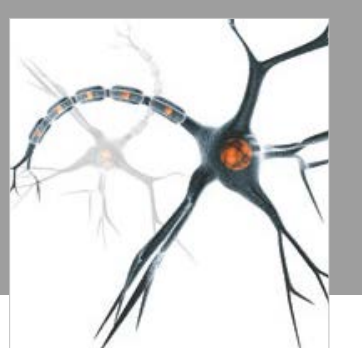

Neural Plasticity

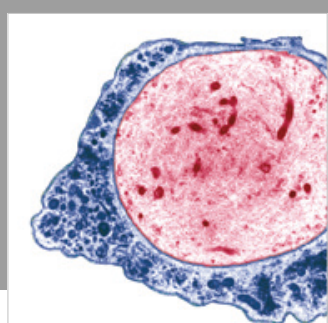

Multiple Sclerosis

International

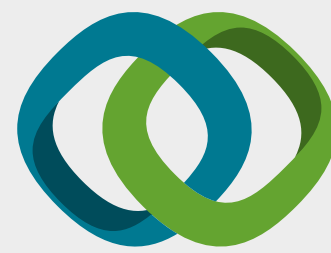

Hindawi

Submit your manuscripts at

www.hindawi.com
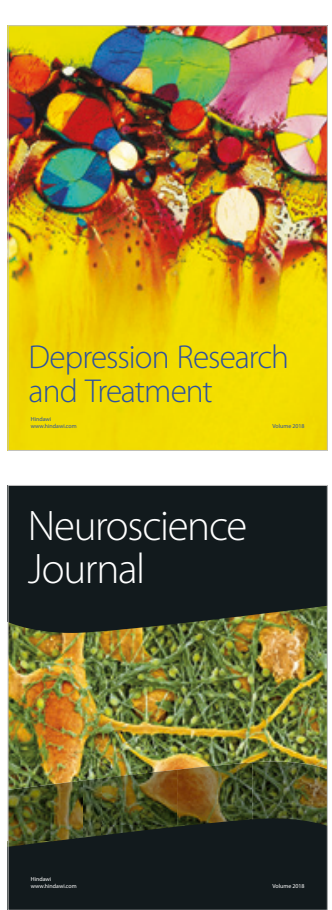

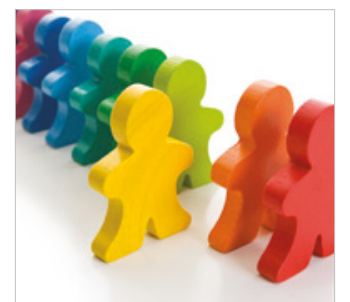

Autism

Research and Treatment
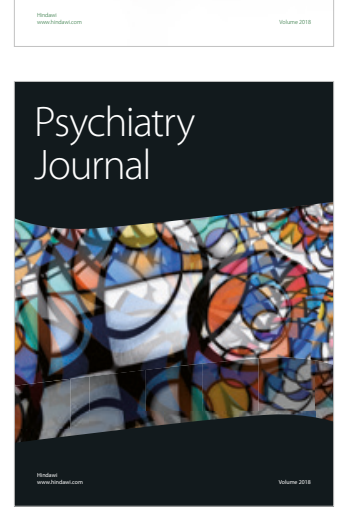
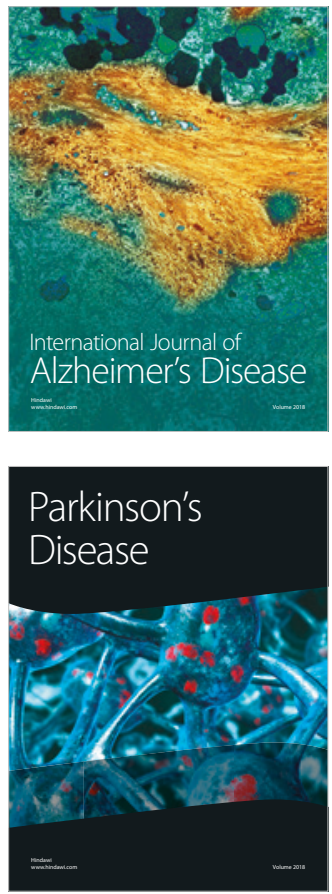
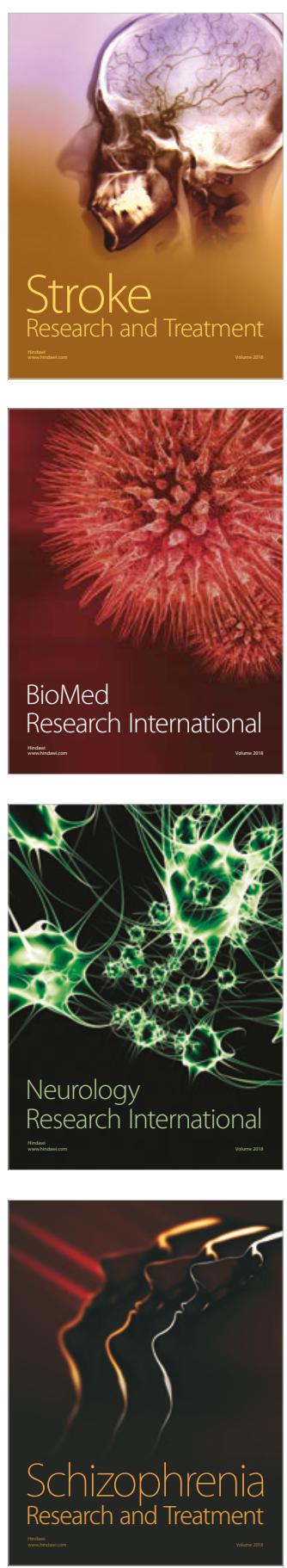